\title{
Quantitative Studies of Membrane Proteins in Whole Cells with Different Methods of Liquid Phase Scanning Transmission Electron Microscopy
}

\author{
$\underline{\text { Diana B. Peckys }}^{1}$, Dalia Alansary ${ }^{1}$, Barbara Niemeyer ${ }^{1}$ and Niels de Jonge ${ }^{2,3}$ \\ 1. Department of Biophysics, Saarland University, 66421 Homburg/Saar, Germany. \\ 2. INM - Leibniz Institute for New Materials, 66123 Saarbrücken, Germany. \\ 3. Department of Physics, University of Saarland, 66123 Saarbrücken, Germany.
}

The majority of today's drugs targets belong to the category of membrane proteins, located at the border between the interior and the outer environment of a cell. Residing in this interfacial position, membrane proteins are responsible for communication and exchange between the individual cell, the neighboring cells, other extracellular structures, and in general with the whole rest of the organism. Studying this crucial group of proteins at the level of single-cells, single-molecules, and in their natural environment, i.e. the plasma membrane, is still a challenge. This is mainly due to a multitude of technical limitations and to the scarcity of available methods. Liquid phase scanning transmission electron microscopy (Liquid STEM) offers new options to explore these proteins in whole cells. Quantitative data from up to hundred thousand of single molecules and dozens of single cells can be gained within only a few days. While well-established, conventional electron microscopy methods for cells involve lengthy preparation procedures of dehydration, staining, embedding and sectioning, and are mostly incompatible for the examination of intact cells, liquid phase STEM involves comparatively fast and non-disruptive preparation steps, much resembling protocols for fluorescence microscopy. Other advantages are the minimized risk of artifacts, and the opportunity to use correlative light and electron microscopy. The latter can be used to detect rare cellular events, or to choose certain cells out of a larger cell population. For the imaging of wet and intact cells, three different methodologies are available: 1) a dedicated liquid flow holder, enclosing the cellular samples, in liquid, in a microfluidic chamber, 2) an environmental scanning electron microscope (ESEM) equipped with an integrated STEM detector, which allows the imaging of wet cells in a saturated water vapor atmosphere, and 3) use of multilayer or single layer graphene sheets to "wrap" and thus to protect the wet cells inside the high vacuum specimen chambers of any conventional STEM [1].

The principle of all Liquid STEM methods relies on the imaging of small, electron dense nanoparticle (NP) labels, specifically coupled to the proteins of interest, and detectable with a spatial resolution of 1-3 nm. The key characteristics of the labeling approaches are 1) a minimized distance between the NP label and its target protein, and 2) a one-to-one stoichiometry between both. Optimized labeling procedures allow to record label position data of hundred thousands individual target proteins. The automatically detected coordinates are subjected to statistical analysis methods, such as the pair correlation function, and algorithms designed to detect cluster sizes or special spatial arrangements. The results allow differentiating between true protein interactions and random proximity, which is the key requirement for any quantitative examination of membrane protein interactions and oligomerization. This capability can be applied, for instance to study drug effects, to explore protein reactions accompanying their activation and functioning, in addition, nanotoxicological NP-cell interactions can be examined [2].

To demonstrate how Liquid STEM methods can answer biological questions, representative recent results will be shown from studies in the field of cancer research, involving the growth factor receptor HER2 and the targeted antibody drug trastuzumab [3], and from fundamental research about the stoichiometry of the ORAI1 ion channel [4], playing an essential role in intracellular $\mathrm{Ca}^{2+}$ homeostasis, indispensible for instance for T-cell activation. In addition, we will also discuss the prospect of imaging live cells $[5,6]$. 


\section{References:}

[1] IN Dahmke et al, ACS Nano 11 (2017), p. 11108.

[2] D Vanhecke et al, Beilstein J Nanotech 8 (2017) p. 2396.

[3] DB Peckys et al, Mol Biol Cell 28 (2017), p. 3193.

[4] DB Peckys et al, Micros Microanal 22 (2016), p. 902.

[5] N de Jonge and DB Peckys, ACS Nano 10 (2016), p. 9061.

[6] The authors thank E. Arzt for his support through INM and the DFG through SFB1027.
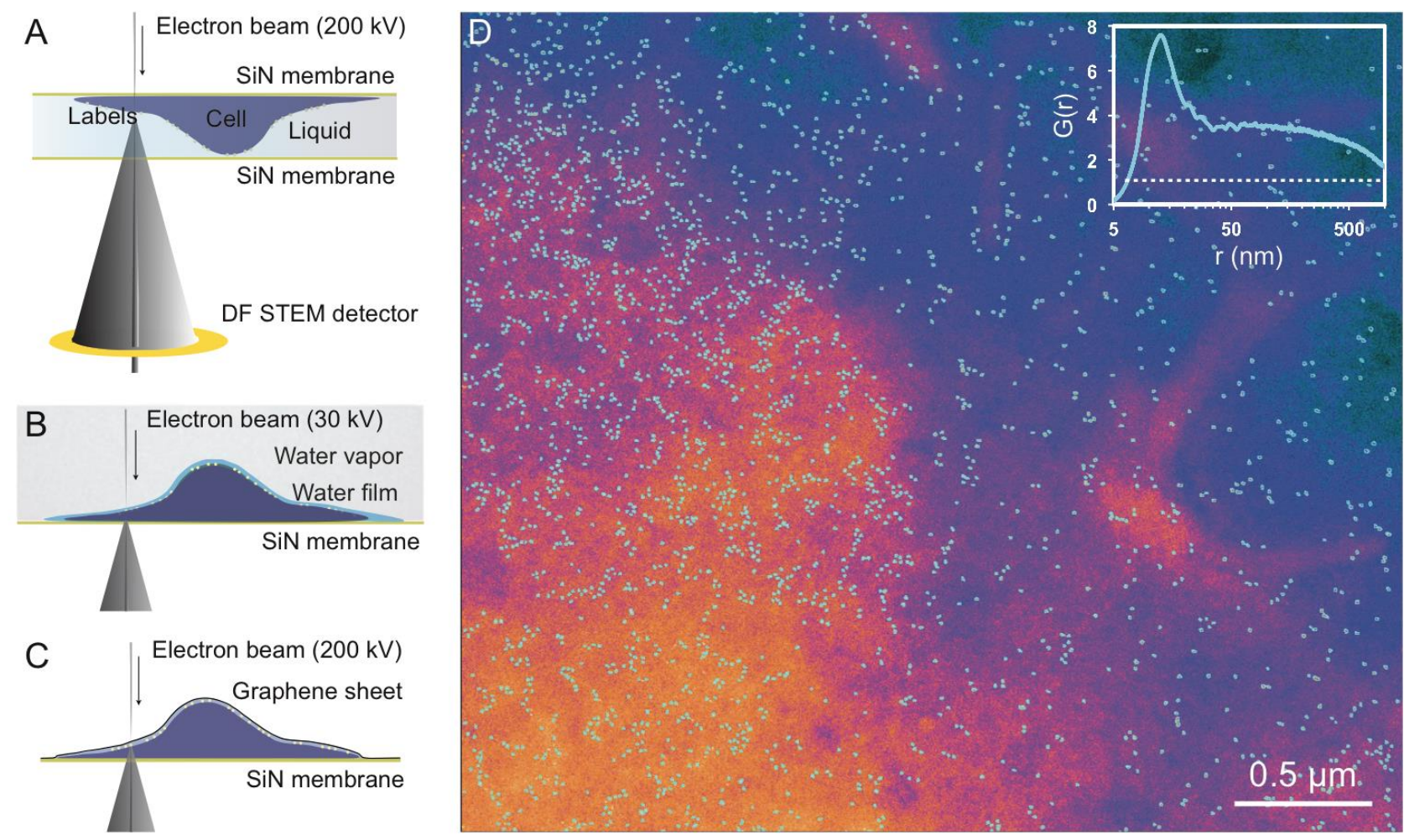

Figure 1. Liquid STEM of labeled membrane proteins in whole mammalian cells using annylar dark field (ADF) detection. (A-C) Schemes of the three types of Liquid STEM for imaging of cellular samples. (A) Dedicated liquid flow holder with the cells enclosed between two silicon nitride (SiN) membranes, (B) ESEM with STEM detector of a cellular sample covered by a thin water layer in in a water vapor environment, and (C) coverage of the cells with a graphene sheet, suitable for any STEM system. (D) STEM image (false colored) recorded from graphene-covered cell expressing HA-tagged ORAI1 proteins, after $\mathrm{Ca}^{2+}$ influx was initiated. QD labels mark the individual ORAI1 channel subunits, clustering of ORAI1 oligomers is seen on the left image side. Inset shows the corresponding pair correlation analysis G(r) of the 2543 labeled ORAI1 molecules, automatically detected in the image, revealing dimerization, oligomerization and larger clustering. 